\title{
Erythema Nodosum Masking Kawasaki Disease with an Initial Manifestation of Skin Lesions
}

\author{
Seigo Okada ${ }^{1}$, Yuichi Ishikawa ${ }^{1}$, Maiko Shimomura ${ }^{1,2}$, Shinpei Sunagawa ${ }^{1,3}$, Reiji Hirano ${ }^{1}$, \\ Shinnosuke Fukunaga ${ }^{1}$, Akiko Miyake ${ }^{1}$, Yusuke Okada ${ }^{1}$, and Takashi Maki ${ }^{1,4}$ \\ ${ }^{1}$ Department of Pediatrics, Saiseikai Shimonoseki General Hospital, Shimonoseki; \\ ${ }^{2}$ Department of Pediatrics, Hiroshima University Graduate School of Biomedical \& Health Sciences, Hiroshima; \\ ${ }^{3}$ Sunagawa Children's Clinic, Onoda; \\ ${ }^{4}$ Department of Pediatrics, National Hospital Organization Kanmon Medical Center, Shimonoseki, Japan.
}

\begin{abstract}
We report the first case demonstrating an association between Kawasaki disease (KD) and erythema nodosum (EN). A 3-year-old girl presented with EN as an initial manifestation of $\mathrm{KD}$. At the initial visit, she showed high fever of $40^{\circ} \mathrm{C}$, injection of the oropharynx, cervical lymphadenopathy, and red-purple cutaneous nodules, particularly on the lower limbs. She complained of severe pain in the neck and cutaneous lesions. Initially, the development of EN was attributed to Salmonella spp infection, which was detected in stool culture. However, the patient did not respond to high-dose ampicillin/sulbactam to which the Salmonella spp is sensitive. Echocardiography performed as screening for fever of unknown origin revealed medium-sized aneurysms of the left anterior descending artery. EN masked the diagnosis of $\mathrm{KD}$, and the patient developed a coronary artery lesion. $\mathrm{KD}$ should be considered in the differential diagnosis of refractory EN in pediatric patients.
\end{abstract}

Key Words: Bacterial infections, exanthema, inflammatory disorders, subcutaneous tissue

\section{INTRODUCTION}

Erythema nodosum (EN) is a delayed-type hypersensitivity reaction that primarily presents as erythematous tender nodules on the shins..$^{1-5}$ Common triggers for EN include infection, drugs, pregnancy, malignancy, and inflammatory conditions, although several cases are idiopathic in nature. ${ }^{1,4,5} \mathrm{EN}$ is rare in the pediatric population., ${ }^{2,3}$ The most common etiology is infection caused by Streptococcus pyogenes, Mycobacterium, Yersinia, atypical bacteria, or Salmonella. ${ }^{1-5}$

Kawasaki disease (KD) is an acute, febrile systemic vasculitis that primarily occurs in infants and young children.-8 $\mathrm{Al}-$

Received: December 5, 2018 Revised: January 6, 2019

Accepted: January 17, 2019

Corresponding author: Seigo Okada, MD, PhD, Department of Pediatrics, Saiseikai Shimonoseki General Hospital, 8-5-1, Yasuoka, Shimonoseki, Yamaguchi 759-6603, Japan.

Tel: 81-83-262-2300, Fax: 81-83-262-2301, E-mail: sokada0901@gmail.com

-The authors have no potential conflicts of interest to disclose.

(C) Copyright: Yonsei University College of Medicine 2019

This is an Open Access article distributed under the terms of the Creative Commons Attribution Non-Commercial License (https://creativecommons.org/licenses/ by-nc/4.0) which permits unrestricted non-commercial use, distribution, and reproduction in any medium, provided the original work is properly cited. though its etiology is unknown, an aberrant innate immune response in predisposed individuals has been proposed as an important contributor to the development of inflammatory vasculitis and the clinical manifestations of $\mathrm{KD},{ }^{6,8}$ Patients with KD primarily present with "polymorphic and nonspecific" skin eruption. ${ }^{7}$ Although the most common form is a diffuse maculopapular eruption, rashes can show various forms, including urticarial exanthema, scarlatiniform rashes, erythroderma, erythema multiforme-like rash, micropustular, and pustular eruptions. ${ }^{7}$ However, EN-like lesions in a patient with KD have not been reported.

Here, we report the first case demonstrating an association between KD and EN. EN masked underlying KD, and the patient developed a coronary artery lesion (CAL).

\section{CASE REPORT}

A 3-year-old girl was referred to our hospital with a 3-day history of high fever and leg swelling. She was born to non-consanguineous healthy parents as a full-term infant. At the initial visit, she showed high fever of $40^{\circ} \mathrm{C}$, injection of the orophar- 
ynx, cervical lymphadenopathy, and red-purple cutaneous nodules, particularly on the lower limbs (Fig. 1A) without gastrointestinal symptoms. She complained of severe pain in the neck and cutaneous lesions. She did not show clinical manifestations of KD, such as conjunctival hyperemia, strawberry tongue, bleeding lips, and erythema at Bacille CalmetteGuérin (BCG) inoculation site. She denied a relevant medication history. Hematological parameters were as follows: leukocyte count, $22.1 \times 10^{9} / \mathrm{L}$ with $74.0 \%$ segmented neutrophils; hemoglobin concentration, $11.5 \mathrm{~g} / \mathrm{dL}$ with $34 \%$ hematocrit; platelet count, $332 \times 10^{9} / \mathrm{L}$; fibrinogen level, $>650 \mathrm{mg} / \mathrm{dL}$; total serum protein, $7.2 \mathrm{~g} / \mathrm{dL}$; serum albumin, $3.8 \mathrm{~g} / \mathrm{dL}$; total bilirubin, $0.5 \mathrm{mg} / \mathrm{dL}$; aspartate aminotransferase, $22 \mathrm{IU} / \mathrm{L}$; alanine aminotransferase, $13 \mathrm{IU} / \mathrm{L}$; C-reactive protein (CRP), $9.6 \mathrm{mg} /$ $\mathrm{dL}$; and sodium, $137 \mathrm{mmol} / \mathrm{L}$. Rapid diagnostic tests for $S$. pyogenes and adenovirus were both negative. Antistreptolysin $\mathrm{O}$ antibody titers were within reference ranges. She was diagnosed with EN following acute pharyngitis, and ampicillin/ sulbactam therapy was initiated intravenously at a dose of 150 $\mathrm{mg} / \mathrm{kg} /$ day. Stool culture obtained upon admission revealed only a slight increase in Salmonella spp [09, non H-d (final report was submitted to us on the 10th day of illness)], which appeared to be the causative antigen for EN. Yersinia spp were negative in the stool culture. Although the Salmonella spp were sensitive to ampicillin/sulbactam, she did not improve
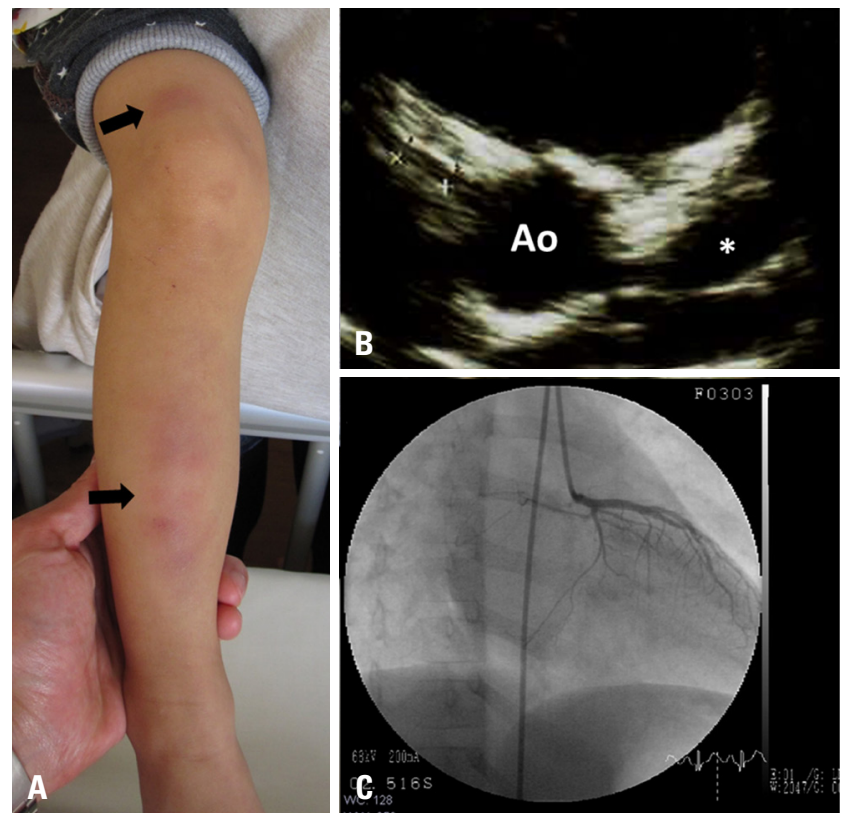

Fig. 1. Patient's cutaneous manifestations upon admission and findings of the coronary artery lesion. (A) Image shows the patient's presentation upon admission (on the 3rd day of illness). Painful, red-purple cutaneous nodules can be observed (arrows), particularly on the lower limbs. (B) A two-dimensional echocardiography scan obtained on the 10th day of illness shows medium-sized aneurysms of the left anterior descending artery, measuring $4.6 \mathrm{~mm}$ (Z: +6.6 , indicated by the asterisk). (C) Selective angiographic image of the left coronary artery obtained during the 2nd month of illness shows that the luminal dimension of the left anterior descending artery has regressed to its normal diameter. Ao, aorta. clinically. On the 5th day of illness, the antibiotic was switched to meropenem at a dose of $100 \mathrm{mg} / \mathrm{kg} /$ day. Following defervescence on the 8th day of illness, the related symptoms including cutaneous lesions disappeared. Echocardiography performed to screen for fever of unknown origin revealed medium-sized aneurysms of the left anterior descending artery [4.6 mm (Z: +6.6)] (Fig. 1B) although it did not show any abnormal findings on admission. This finding led to a diagnosis of incomplete $\mathrm{KD}$, and oral aspirin therapy was initiated. She did not show recurrence of fever, her CRP turned negative on the 17th day of illness, and she was discharged. She did not show any gastrointestinal symptoms during hospitalization. Paired serum antibody of Mycoplasma pneumoniae obtained during the hospitalization were both negative. The CAL gradually regressed after discharge. Skin desquamation was not observed during follow-up. Cardiac catheterization performed 2 months after discharge showed complete regression of the CAL (Fig. 1C). Oral aspirin therapy was discontinued after the cardiac catheterization. To date, follow-up echocardiogram has determined no apparent CAL without any cardiovascular events. Written informed consent was obtained from the patient's parents.

\section{DISCUSSION}

This is the first case report to describe a patient with $\mathrm{KD}$ who presented with EN as the initial manifestation. EN-related symptoms masked those of KD with a consequent delay in the diagnosis of $\mathrm{KD}$ and the development of CAL. The CAL regressed spontaneously without any subsequent cardiovascular events in the patient.

The pathogenesis of EN was a major concern in our patient. Initially, the development of EN was attributed to Salmonella spp infection. ${ }^{1-5}$ However, stool culture showed only slight bacterial growth, and the patient showed no gastrointestinal symptoms. Moreover, the patient did not respond to highdose ampicillin/sulbactam to which the Salmonella spp is sensitive. Based on these findings, the development of EN was considered a component of the major manifestations of KD and not a sequela of Salmonella infection. Although EN is histopathologically characterized by septal panniculitis without vasculitis, ${ }^{1,2}$ it might occur in patients with $\mathrm{KD}$ because skin lesions in $\mathrm{KD}$ are typically described as "nonspecific."7-9 Moreover, not all symptoms related to KD are explained by vasculitis alone. ${ }^{9,10}$ We did not perform a skin biopsy in our patient, which serves as a limitation of this study.

Another concern is that the clinical presentation of EN and $\mathrm{KD}$ is indistinguishable. In the present case, prolonged fever, injection of the oropharynx, cervical lymphadenopathy, neck pain, rash, and swelling of the extremities could be observed as features of both conditions. ${ }^{1-5,7,8}$ Conjunctival hyperemia, strawberry tongue, bleeding lips, and/or erythema at the BCG 
inoculation site could be helpful to distinguish KD from EN; however, these findings might not always be observed in older patients. ${ }^{11}$

EN in the pediatric population is rare; however, it may occur following various conditions, including KD. KD should be considered in the differential diagnosis of refractory EN in pediatric patients.

\section{AUTHOR CONTRIBUTIONS}

Conceptualization: Seigo Okada. Data curation: Seigo Okada, Yuichi Ishikawa, Maiko Shimomura, Shinpei Sunagawa, Akiko Miyake, Yusuke Okada. Formal analysis: Seigo Okada, Yuichi Ishikawa. Investigation: Seigo Okada. Methodology: Seigo Okada, Reiji Hirano, Shinnosuke Fukunaga. Project administration: Seigo Okada. Software: Seigo Okada, Yuichi Ishikawa. Supervision: Reiji Hirano, Shinnosuke Fukunaga, Takashi Maki. Validation: Takashi Maki. Visualization: Seigo Okada. Writing—original draft: Seigo Okada. Writing—review \& editing: Reiji Hirano, Shinnosuke Fukunaga, Takashi Maki.

\section{ORCID iDs}

Seigo Okada

Yuichi Ishikawa

Maiko Shimomura

Shinpei Sunagawa

Reiji Hirano

Shinnosuke Fukunaga

Akiko Miyake

Yusuke Okada

Takashi Maki

\section{REFERENCES}

https://orcid.org/0000-0002-9150-1913 https://orcid.org/0000-0003-0470-7807 https://orcid.org/0000-0002-8064-7677 https://orcid.org/0000-0001-6963-1116 https://orcid.org/0000-0003-1767-2906 https://orcid.org/0000-0001-8677-2076 https://orcid.org/0000-0002-0816-6748 https://orcid.org/0000-0001-8173-1224 https://orcid.org/0000-0002-2143-3783

1. Ozbagcivan O, Akarsu S, Avci C, Inci BB, Fetil E. Examination of the microbial spectrum in the etiology of erythema nodosum: a retrospective descriptive study. J Immunol Res 2017;2017:8139591.

2. Aydın-Teke T, Tanır G, Bayhan GI, Metin O, Oz N. Erythema nodosum in children: evaluation of 39 patients. Turk J Pediatr 2014; 56:144-9.

3. Kakourou T, Drosatou P, Psychou F, Aroni K, Nicolaidou P. Erythema nodosum in children: a prospective study. J Am Acad Dermatol 2001;44:17-21.

4. Cribier B, Caille A, Heid E, Grosshans E. Erythema nodosum and associated diseases. A study of 129 cases. Int J Dermatol 1998;37: 667-72.

5. Psychos DN, Voulgari PV, Skopouli FN, Drosos AA, Moutsopoulos HM. Erythema nodosum: the underlying conditions. Clin Rheumatol 2000;19:212-6.

6. Hara T, Nakashima Y, Sakai Y, Nishio H, Motomura Y, Yamasaki S. Kawasaki disease: a matter of innate immunity. Clin Exp Immunol 2016;186:134-43.

7. McCrindle BW, Rowley AH, Newburger JW, Burns JC, Bolger AF, Gewitz M, et al; American Heart Association Rheumatic Fever, Endocarditis, and Kawasaki Disease Committee of the Council on Cardiovascular Disease in the Young; Council on Cardiovascular and Stroke Nursing; Council on Cardiovascular Surgery and Anesthesia; and Council on Epidemiology and Prevention. Diagnosis, treatment, and long-term management of Kawasaki disease: a scientific statement for health professionals from the American Heart Association. Circulation 2017;135:e927-99.

8. Kim KY, Kim DS. Recent advances in Kawasaki disease. Yonsei Med J 2016;57:15-21.

9. Ming A, Wargon O. Annular lesions in Kawasaki disease: a cause of confusion. Australas J Dermatol 2008;49:207-12.

10. Okada S, Kobayashi-Fujiwara Y, Oga A, Furuta T, Ikemoto K, Fujii $\mathrm{H}$, et al. Distinct distribution of immunocytes in a retropharyngeal lymphadenopathy associated with Kawasaki disease: a case study compared with tonsillitis. Cardiology 2017;137:237-43.

11. Cai Z, Zuo R, Liu Y. Characteristics of Kawasaki disease in older children. Clin Pediatr (Phila) 2011;50:952-6. 